\title{
1 Chromatin accessibility of primary human cancers ties regional mutational 2 processes with tissues of origin
}

4 Oliver Ocsenas ${ }^{1,2}$, Jüri Reimand $1,2,3, @$

6 1. Computational Biology Program, Ontario Institute for Cancer Research, Toronto, ON, Canada

7 2. Department of Medical Biophysics, University of Toronto, Toronto, ON, Canada

8 3. Department of Molecular Genetics, University of Toronto, Toronto, ON, Canada

9 @ correspondence: Juri.Reimand@utoronto.ca

\section{ABSTRACT:}

12 Regional mutagenesis in cancer genomes associates with DNA replication timing (RT) and

13 chromatin accessibility (CA) of normal cells, however human cancer epigenomes remain

14 uncharacterized in this context. Here we model megabase-scale mutation frequencies in 2517

15 cancer genomes with $773 \mathrm{CA}$ and RT profiles of cancers and normal cells. We find that CA

16 profiles of matching cancers, rather than normal cells, predict regional mutagenesis and

17 mutational signatures, indicating that most passenger mutations follow the epigenetic landscapes

18 of transformed cells. Carcinogen-induced and unannotated signatures show the strongest

19 associations with epigenomes. Associations with normal cells in melanomas, lymphomas and

20 SBS1 signatures suggest earlier occurrence of mutations in cancer evolution. Frequently mutated

21 regions unexplained by CA and RT are enriched in cancer genes and developmental pathways,

22 reflecting contributions of localized mutagenesis and positive selection. These results underline

23 the complex interplay of mutational processes, genome function and evolution in cancer and

24 tissues of origin. 


\section{INTRODUCTION}

26

27

The cancer genome is a footprint of its evolution and molecular environment that is shaped by somatic mutations, such as single nucleotide variants (SNVs) and structural alterations $(1,2)$. While a minority of mutations called drivers confer cells with selective advantages (3-5), most mutations are considered functionally neutral passengers that are caused by diverse mutational processes (6-8). Somatic mutagenesis and positive selection of known cancer genes also affects normal tissues $(9,10)$. Characterizing the landscape of somatic mutations helps understand the underlying mutational processes and better evaluate the functional consequences of mutations and their roles in cancer etiology and evolution.

Processes of somatic mutagenesis act at different scales of the genome $(11,12)$. At the trinucleotide resolution, mutational signatures of SNVs are associated with endogenous and exogeneous processes related to aging, carcinogen exposures, DNA repair deficiencies, and cancer therapies $(6,13)$. At the local resolution of 100-1000 bps, non-coding genomic elements, such as transcription start sites and binding sites of CTCF, are enriched in mutations (14-16). However, the precise molecular mechanisms driving these mutational processes remain uncharacterized. At the regional, megabase-scale resolution of the genome, variation in mutation frequencies shows a complex interplay of DNA replication timing (RT), chromatin accessibility (CA) and transcriptional activity (17-19). Early-replicating, transcriptionally active regions of open chromatin have fewer mutations than late-replicating, passive regions of heterochromatin, potentially due to increased error rates and decreased mismatch repair later in DNA replication (20-23). Mutational signatures are distributed asymmetrically with respect to DNA replication origins and timing (24). Regional mutagenesis has been associated with epigenetic information of related normal cells, providing evidence of cells of cancer origin contributing to somatic variation (25) and allowing classification of cancers of unknown origin (26). However, CA and RT profiles of only common cell lines and normal tissues have been used to characterize regional mutational processes while the epigenetic landscapes of primary human cancers remain unexplored.

To decipher regional mutational processes in the context of cancer epigenomes, we analyzed a large and diverse collection of CA and RT profiles of cancers, normal tissues, and cell lines as predictors of regional mutagenesis in thousands of whole cancer genomes using machine learning. CA profiles of matching cancer types, rather than normal tissues, appear as determinants of regional mutagenesis and mutational signatures. We found tissue-of-origin effects of CA and RT in most predictions, bespoke deviations in specific cancer types and mutational signatures, and a convergence of excess mutations to developmental and cancer pathways. Together, these results underline the spatial and temporal complexity of regional mutagenesis in cancer genomes. 


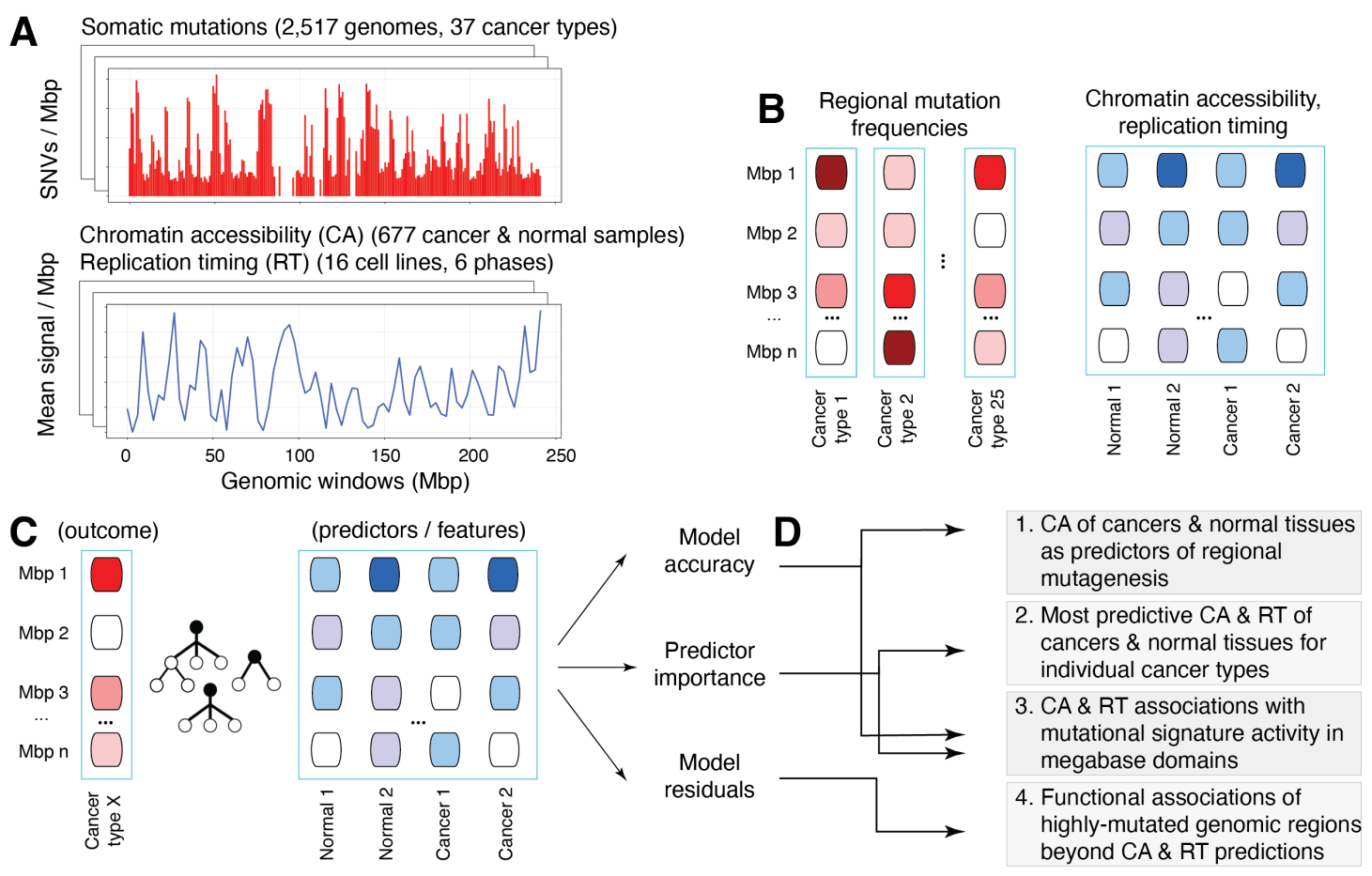

Figure 1. Characterizing chromatin accessibility (CA) and replication timing (RT) as determinants of regional mutagenesis in cancer genomes. A. Somatic mutations in cancer genomes (top) and CA and RT datasets of normal tissues and cancers (bottom) were integrated to study regional mutational processes. Somatic single nucleotide variants (SNVs) of 2,517 whole cancer genomes of the PCAWG project were analyzed with 677 genome-wide CA profiles of primary human cancers, normal tissues and cell lines, and 96 RT profiles of cell lines and cell cycle phases. B. SNVs were aggregated by summing in 2,465 high-confidence genomic regions of one megabase (Mbp) as a measure of regional mutagenesis. Mean CA and RT scores per Mbp were derived for all profiles. C. Random forest models were trained using regional mutagenesis data as the outcome and CA and RT profiles as predictors. 25 cancer types of the largest cohorts in PCAWG and the pan-cancer cohort were analyzed. D. To associate regional mutagenesis with $\mathrm{CA}$ and $\mathrm{RT}$, mutational signatures and gene function, models were evaluated in terms of accuracy, predictor importance, and model residuals.

\section{RESULTS}

\section{Chromatin accessibility of primary cancers is a major determinant of regional mutagenesis}

64 To evaluate the associations of CA and RT with regional mutagenesis in cancer genomes, we analyzed somatic variant calls of whole cancer genomes, $677 \mathrm{CA}$ profiles of primary human cancers, normal tissues and cell lines, and RT profiles of 16 cell lines in 6 cell cycle phases using the random forest framework (Figure 1). We integrated 23 million SNVs from 2,517 whole cancer genomes spanning 37 types of the ICGC/TCGA PCAWG project (1) with CA and RT profiles collected from The Cancer Genome Atlas (TCGA), Epigenomics Roadmap and ENCODE3 projects (27-30) (Supplementary Figures 1-2). Focusing on 2,465 mappable onemegabase regions, we derived somatic SNV counts for the pan-cancer dataset and 25 cancer types of the largest cohorts. We processed $773 \mathrm{CA}$ and RT profiles of primary cancers, normal tissues, and cell lines as mean genomic signals per megabase (Figure 1B). To map the complex non-linear associations of $\mathrm{CA}$ and $\mathrm{RT}$ with regional mutagenesis, random forest regression 
models were trained with megabase-scale mutation frequencies as outcomes and CA and RT profiles as predictors (i.e., features) (Figure 1C). The most informative predictors were quantified statistically and using local prioritization methods (31) (Figure 1D). As expected, late

78 RT profiles inversely correlated with CA profiles and regional mutagenesis profiles clustered according to cancer types (Supplementary Figures 3-4).

80 Given the diverse collection of epigenomic profiles, we asked whether CA profiles of cancers (n

$81=383)$ or normal cells and tissues $(n=244)$ were more informative of regional mutagenesis in

82 cancer genomes. We predicted regional mutation frequencies using random forests in two

83 Monte-Carlo cross-validation experiments with matched data splits where the predictors included

84 either cancer CA profiles or normal CA profiles, respectively. RT profiles were also included in

85 both models to focus on the relative contributions of CA profiles. We found that CA profiles of

86 cancers were more accurate predictors of regional mutagenesis in 19/25 cancer types (empirical

$87 P<0.01$ ) (Figure 2A). The strongest signal was observed in breast cancer where the predictions

88 informed by cancer CA profiles were nearly twice as accurate as the models informed by normal

89 tissue CA (median adj. $R^{2} 0.69$ vs. $0.36 ; P<0.001$ ) (Figure 2B). Stronger associations of cancer

90 CA profiles and regional mutagenesis were also found in cancers of the prostate, ovary, uterus,

91 kidney, and pancreas $\left(\Delta\right.$ adj. $\left.\mathrm{R}^{2}>0.1 ; P<0.001\right)$ and in the pooled pan-cancer set of 37 cancer

92 types (adj. $\mathrm{R}^{2} 0.90$ vs. $0.87 ; P<0.001$ ). A few exceptions were also apparent; in melanoma,

93 models utilizing CA profiles of normal tissues were significantly more accurate (adj. $\mathrm{R}^{2} 0.69$ for

94 normal CA vs. 0.65 cancer CA; $P=0.004$ ). The high somatic mutation burden of normal skin

95 cells due to long-term ultraviolet light exposure (9) appears consistent with the model predictions

96 that a fraction of mutations in melanomas are distributed according to CA profiles of normal

97 tissues. In medulloblastoma, CA profiles of normal tissues were also more predictive of regional

98 mutagenesis (adj. $\mathrm{R}^{2}=0.45$ for normal $\mathrm{CA} v s$. adj. $\mathrm{R}^{2}=0.36$ for cancer $\mathrm{CA} ; P=0.001$ ),

99 potentially explained by the developmental origin of this pediatric brain cancer (32). CA profiles

100 of normal tissues also improved prediction accuracy in B-cell non-Hodgkin's lymphoma

101 (BNHL) and chronic lymphocytic leukemia (CLL) $(P<0.01)$. Overall model accuracy was

102 partially explained by genome-wide mutation burden of cancer types (Spearman rho $=0.61, P=$

1030.0011 ) but not cohort size (rho $=0.22, P=0.28$ ) (Supplementary Figure 5). In summary,

104 regional mutagenesis is more strongly associated with CA of primary human cancers rather than

105 normal tissues and cell lines in most cancer types, indicating that most somatic mutations occur

106 after the cells have acquired the epigenetic characteristics of cancer cells. 
bioRxiv preprint doi: https://doi.org/10.1101/2021.05.14.444202; this version posted May 17, 2021. The copyright holder for this preprint (which was not certified by peer review) is the author/funder, who has granted bioRxiv a license to display the preprint in perpetuity. It is made available under aCC-BY 4.0 International license.
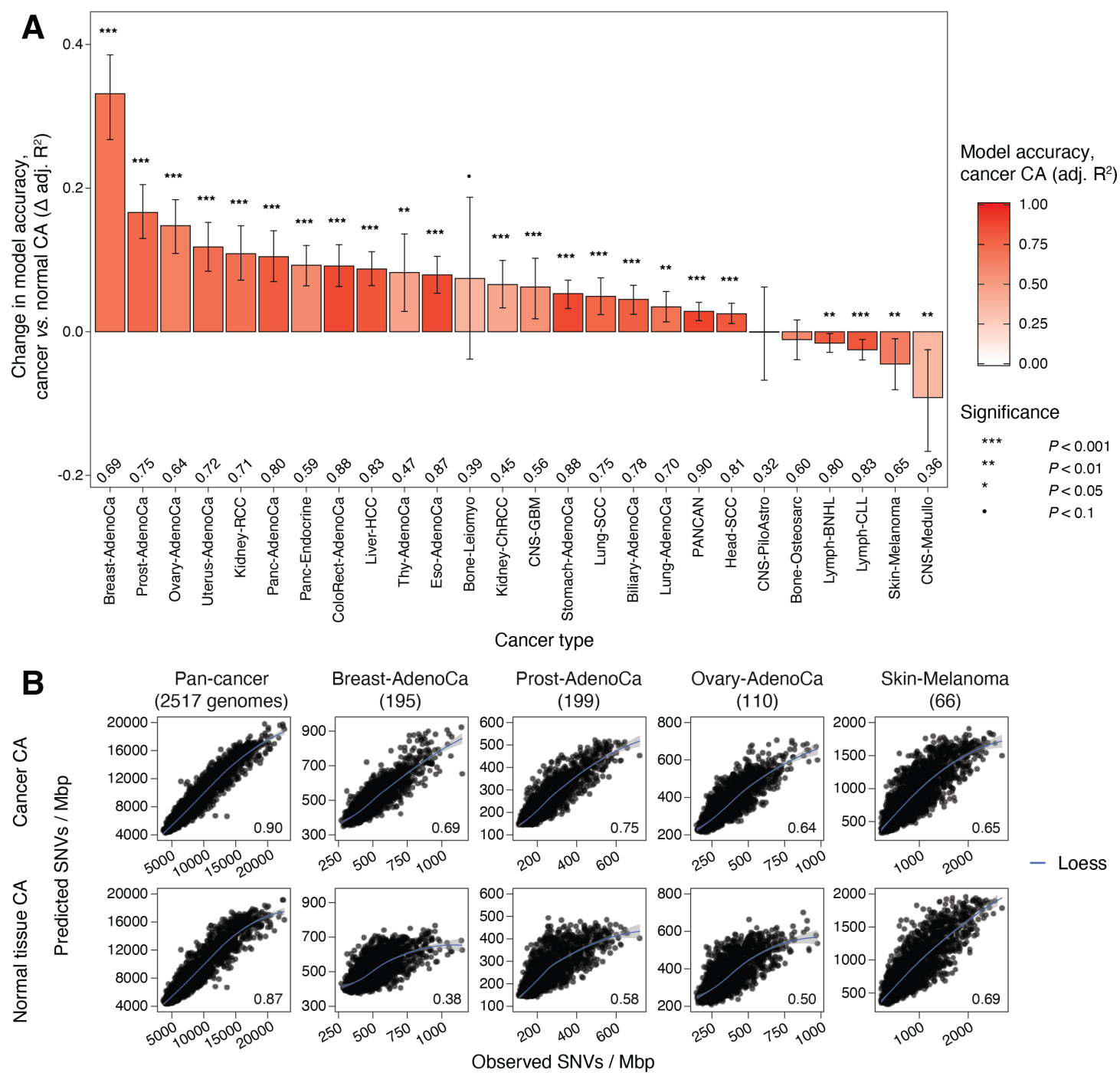

Figure 2. Chromatin accessibility of primary cancers is a major determinant of regional mutagenesis in cancer genomes. A. Random forest models informed by CA profiles of primary cancers are more accurate predictors of regional mutagenesis compared to models informed by normal tissues. Bar plot shows relative changes in accuracy $\left(\Delta\right.$ adjusted $\mathrm{R}^{2}$ ) of cancer CA-informed models in 25 cancer types in PCAWG. Replication timing (RT) profiles are included in both classes of models as reference. Permutation P-values and $95 \%$ bootstrap confidence intervals are shown. Accuracy of models informed by cancer CA profiles are shown below bars (adj. $R^{2}$ ). B. Examples of regional mutagenesis predicted using CA profiles of cancers (top) and normal tissues (bottom). Scatterplots show observed and model-predicted mutation frequencies. Model accuracy values are shown below. 
bioRxiv preprint doi: https://doi.org/10.1101/2021.05.14.444202; this version posted May 17, 2021. The copyright holder for this preprint (which was not certified by peer review) is the author/funder, who has granted bioRxiv a license to display the preprint in perpetuity. It is made available under aCC-BY 4.0 International license.

A

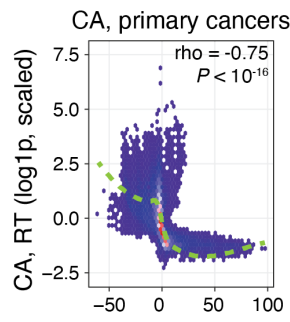

$\mathrm{CA}$, tissues \& cell lines $\mathrm{RT}$, early $(\mathrm{G} 1, \mathrm{~S} 1, \mathrm{~S} 2)$
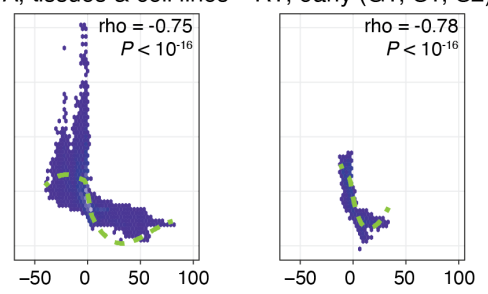

$\mathrm{RT}$, late (S3, S4, G2)
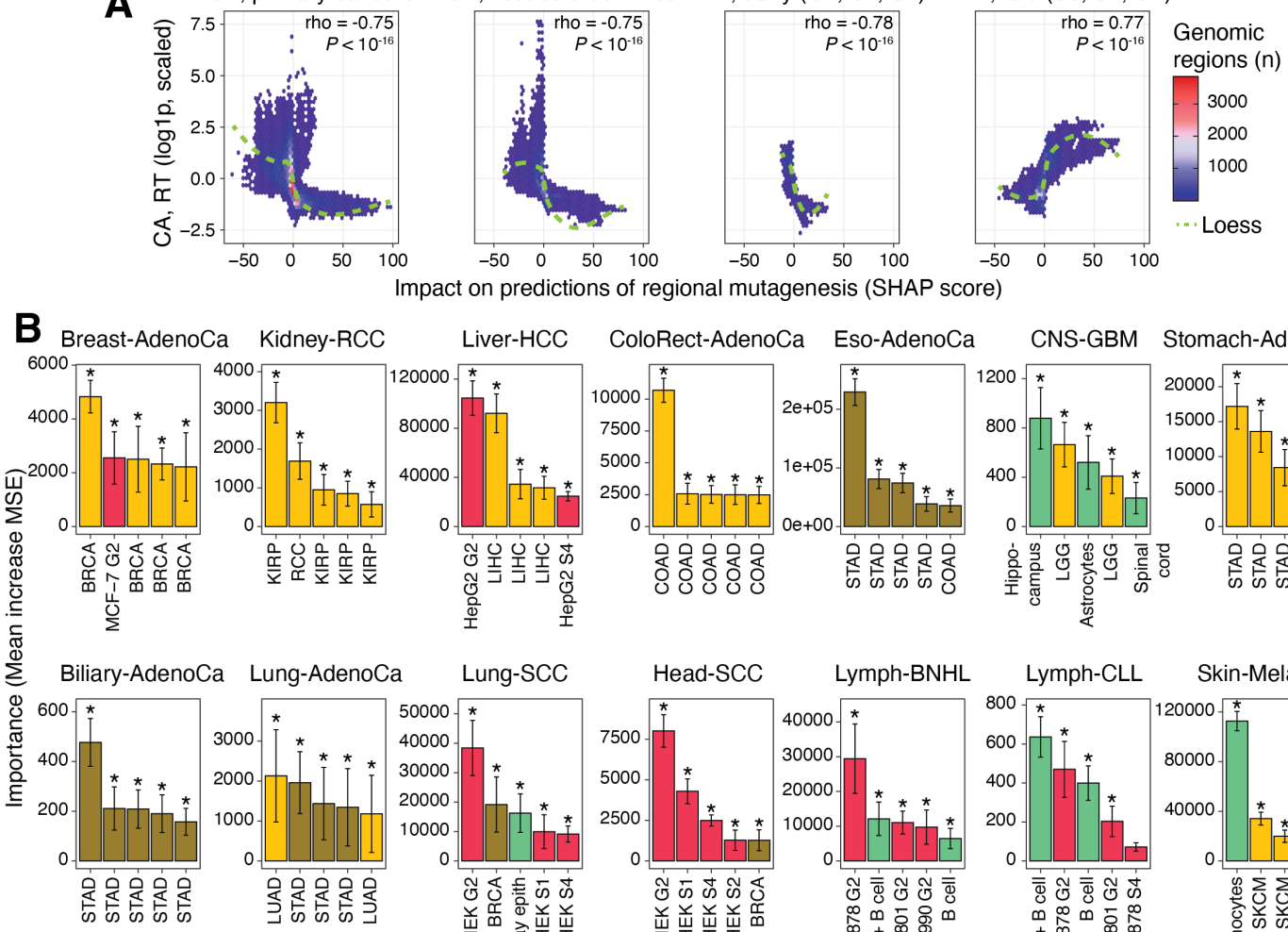

Impact on predictions of regional mutagenesis (SHAP score)

Liver-HCC ColoRect-AdenoCa Eso-AdenoCa

CNS-GBM Stomach-AdenoCa
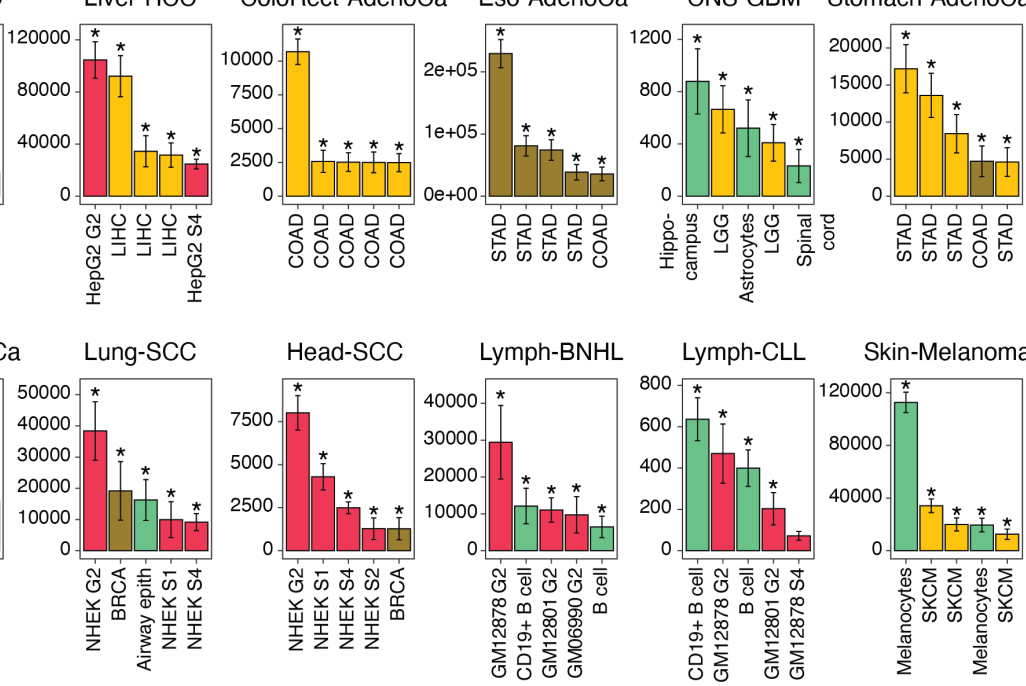

Predictors of regional mutagenesis

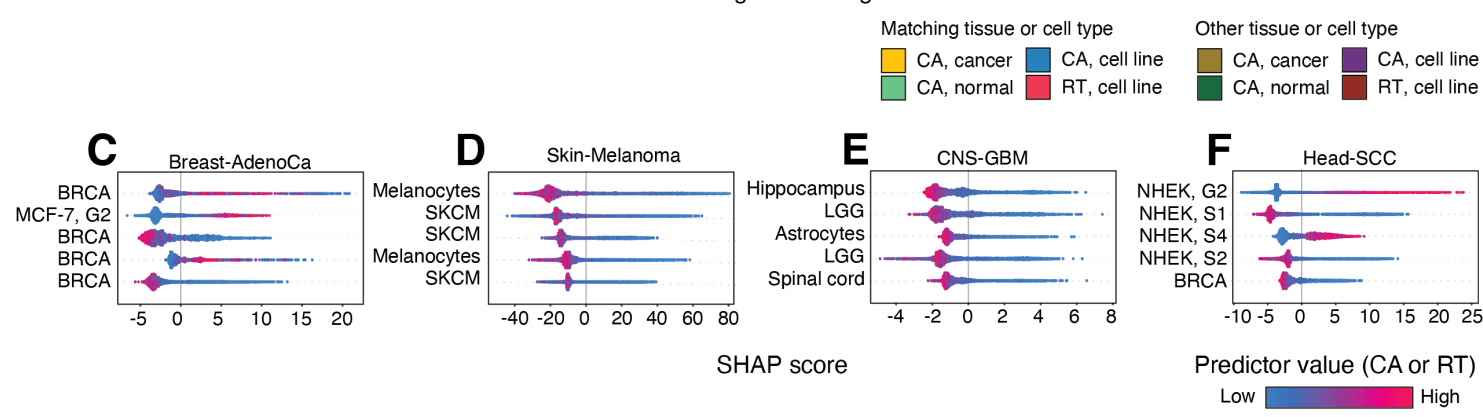

Figure 3. Top predictors of regional mutagenesis tie chromatin accessibility and replication timing with sites of cancer origin. A. Negative associations of CA and positive associations of late RT with regional mutagenesis are found in local predictor analysis of random forest models. 2D density plots summarize the effects of individual CA and RT profiles (Y-axis) on regional mutagenesis across 14 cancer types. The five most significant predictors of 14 cancer types are quantified using Shapley additive explanation (SHAP) scores (X-axis). Spearman correlation values are shown (top right). B. Major predictors of regional mutagenesis represent cancer tissues of origin. Barplot shows importance scores of top-5 predictors in random forest models of 14 cancer types $(P<0.001$; $\pm 1 \mathrm{~s}$.d.). Colors indicate the predictor type (CA, RT) and its relationship to the cancer type where mutagenesis is predicted (matching or other). Brighter colors indicate predictors that match cancer type or tissues or cells of origin. C-F. Examples of predictors of regional mutagenesis. SHAP scores show the impact of a predictor on the predictions (X-axis) and corresponding predictor values (color gradient). C. Top predictors in breast cancer include four CA profiles of primary breast cancers (BRCA) and one RT profile of the breast cancer cell line MCF-7 in phase G2 of cell cycle. D. Top predictors in melanoma include the CA profiles of normal melanocytes and melanomas (SKCM). E. Top predictors in GBM include CA profiles of lower-grade gliomas (LGG) and normal neuronal tissues. F. Top predictors in head squamous cell carcinoma include RT profiles of the squamous cell line NHEK (primary normal human epidermal keratinocytes) and a CA profile of BRCA 


\section{Top predictors of regional mutagenesis match cancer types and sites of origin}

111 To interpret regional mutagenesis through cancer tissues of origin, we asked which CA and RT

112 profiles contributed the most to the predictive models. We included all 773 profiles as predictors

113 and analyzed 14 cancer types for which profiles of primary cancers and relevant normal tissues

114 were available. We selected five most significant predictors for each cancer type $(P<0.001)$ and

115 quantified these using Shapley Additive exPlanation (SHAP) scores (31) that reflect associations

116 with mutation burden accumulated across genomic regions. SHAP scores were negatively

117 correlated with CA of cancers and normal tissues (rho $=-0.75 ; P<10^{-16}$ ) (Figure 3A). Late-

118 replicating regions were positively correlated with regional mutagenesis (rho $=0.77, P<10^{-16}$ )

119 while early replicating regions showed a less-variable negative correlation (rho $=-0.78, P<10^{-}$

$120{ }^{16}$ ). This inverse relationship of CA and RT with respect to regional mutagenesis is consistent

121 with previous studies (17-23), however it is extended to a diverse collection of CA and RT

122 profiles of primary cancers, normal tissues, and cell lines. Non-linear associations of regional

123 mutagenesis and its epigenomic predictors are apparent in individual cancer types

124 (Supplementary Figure 6). This analysis underlines the complex interactions of regional

125 mutagenesis with CA and RT in this pan-cancer cohort and warrants detailed analysis of

126 individual predictors.

127 We examined the top predictors of regional mutagenesis. CA profiles of matching cancers were

128 among the strongest predictors in eight of 14 cancer types including liver, breast, kidney,

129 stomach, and colorectal cancers $(P<0.001)$, emphasizing tissue-of-origin associations (Figure

130 3B). For example, regional mutagenesis in breast cancer showed positive and negative

131 associations with four CA profiles of breast cancers of the TCGA dataset (Figure 3C).

132 Additional associations appeared at the level of organ systems as CA profiles of stomach and

133 colorectal cancers were the top predictors of regional mutagenesis in colorectal, stomach, biliary

134 and esophageal cancers, suggesting similarities of mutational processes or epigenomes of the

135 gastrointestinal tract. Interestingly, regional mutagenesis in lung adenocarcinomas was also

136 explained by CA profiles of stomach and lung adenocarcinomas. Overall, matched cancer-

137 specific CA profiles showed stronger associations with regional mutagenesis than profiles of

138 normal cells.

139 CA profiles of matching normal tissues associated with regional mutagenesis in five cancer

140 types. Mutations in melanoma were predicted by two CA profiles of normal melanocytes and

141 three profiles of melanomas, whereas a three-fold higher feature importance score was assigned

142 to the normal tissue (incMSE $1.2 \times 10^{5}$ vs. $3.4 \times 10^{4}$ ) (Figure 3B). Accessible chromatin of

143 cancers and normal melanocytes was relatively depleted in mutations according to SHAP

144 analysis (Figure 3D). This is consistent with the shaping of melanoma genomes through the

145 chromatin landscape of normal melanocytes (9) earlier in cancer evolution. CA profiles of

146 normal B-cells were found as predictors in lymphoid cancers BNHL and CLL. Somatic

147 hypermutation (SHM) of immunoglobulin genes in normal B-cells and aberrant SHM in

148 lymphomas (33) potentially explains this association. In glioblastoma (GBM), CA profiles of 
149 neuronal tissues (hippocampus, astrocytes, spinal cord) as well as lower-grade gliomas were

150 selected as top features (Figure 3E). The mixture of CA of cancers and normal neural cells

151 predictive of regional mutagenesis of GBM may reflect its extensive intratumoral heterogeneity

152 and proposed origin in stem-like cells (34).

153 In most cases, CA negatively correlated with regional mutagenesis according to SHAP scores,

154 both in CA profiles of primary cancers, such as liver and breast cancer, as well as related normal

155 tissues, such as melanocytes in melanoma and astrocytes in glioma (Figure 3D-E). However,

156 associations of high CA and increased mutation burden were also apparent. In breast cancer, the

157 two most predictive CA profiles showed positive SHAP scores in highly accessible genomic

158 regions, indicating the activity of a mutational process targeting open chromatin (Figure 3C)

159 (Supplementary Figure 6). Thus, deconvoluting the bulk profiles of megabase-scale mutation

160 burden helps map interactions of regional mutagenesis with CA and RT.

161 RT profiles were the major predictors of regional mutagenesis in six cancer types. Mutations in

162 lung and head squamous cell carcinomas (SCC) associated with RT profiles of the squamous cell

163 line of normal human epidermal keratinocytes (NHEK) (Figure 3B,F). The squamous cell

164 association indicates cell-of-origin patterns of regional mutagenesis, while the association with

165 normal cells may reflect mutagenesis earlier in cancer evolution, potentially through the tobacco

166 signature SBS4 that represents $44 \%$ and $12 \%$ of SNVs in the Lung-SCC and Head-SCC cohorts,

167 respectively. Similarly, RT profiles of lymphoblastoid cell lines were among the top predictors in

168 CLL and BNHL. Tissue-specific RT profiles of the cancer cell lines MCF-7 and HepG2 were

169 found in breast and liver cancers, respectively. Most RT predictors (13/16) represented late-

170 replicating cell cycle phases G2 and S4. Individual RT profiles positively associated with

171 mutagenesis in late-replicating regions (e.g., phase $\mathrm{G} 2$ of MCF-7 in breast cancer) and negatively

172 in early-replicating regions (e.g., phase S1 of HNEK in head SCC) (Figure 3C,F), consistent

173 with earlier observations that elevated regional mutagenesis is an effect of increased DNA

174 damage and decreased repair in late replication (20). However, RT profiles were generally

175 underrepresented among top predictors compared to CA profiles. This is likely because fewer

176 and less-diverse RT profiles of cell lines offer only a limited representation of mutational

177 processes in diverse cancer genomes, while CA profiles of primary human cancers provide

178 complementary information. Together, this analysis extends our findings of tissue-specific CA

179 and RT profiles as the principal predictors of regional mutagenesis and underlines cell-of-origin

180 effects and cancer heterogeneity. 
bioRxiv preprint doi: https://doi.org/10.1101/2021.05.14.444202; this version posted May 17, 2021. The copyright holder for this preprint (which was not certified by peer review) is the author/funder, who has granted bioRxiv a license to display the preprint in perpetuity. It is made available under aCC-BY 4.0 International license.
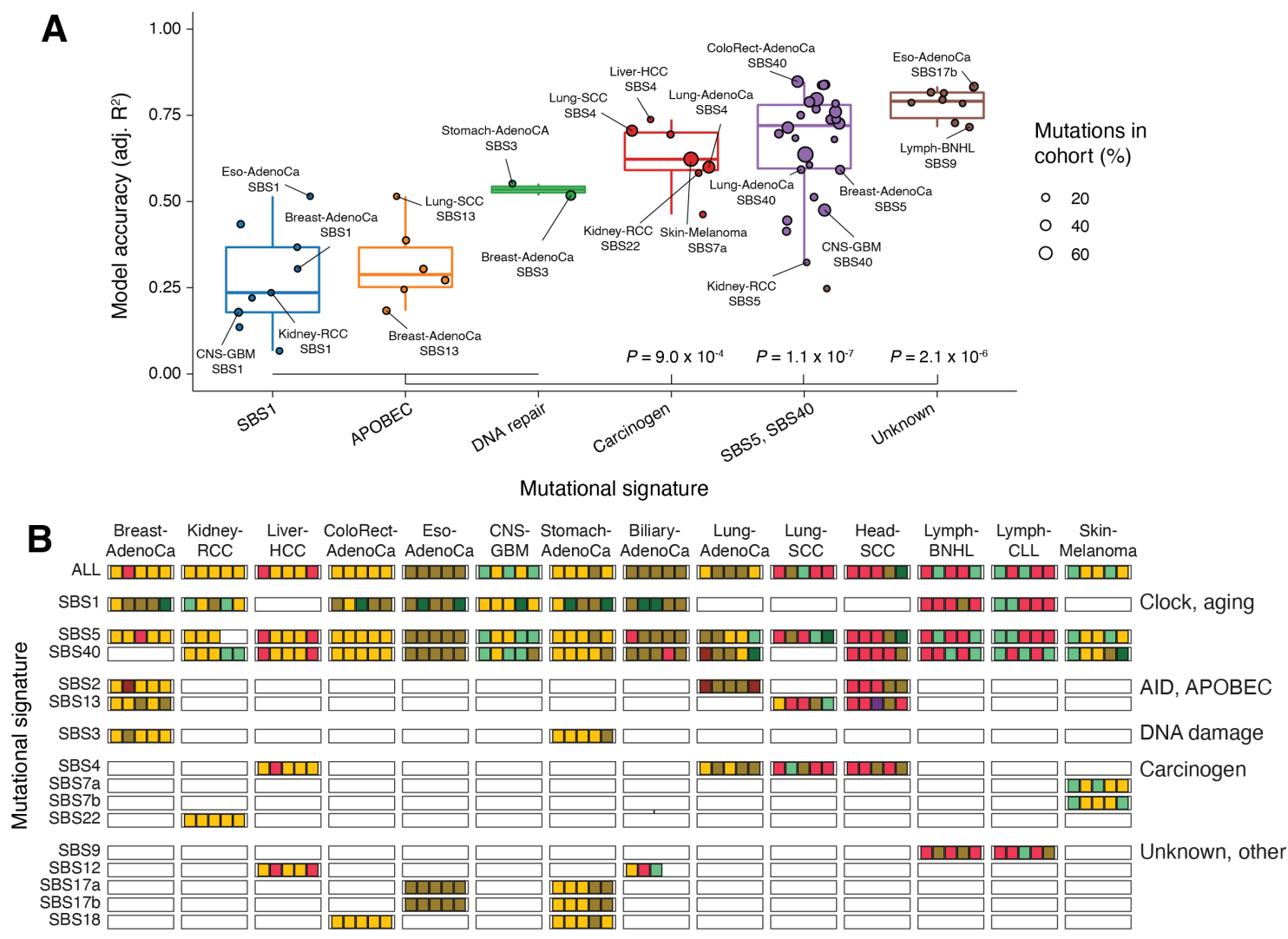

Predictors of mutational signatures
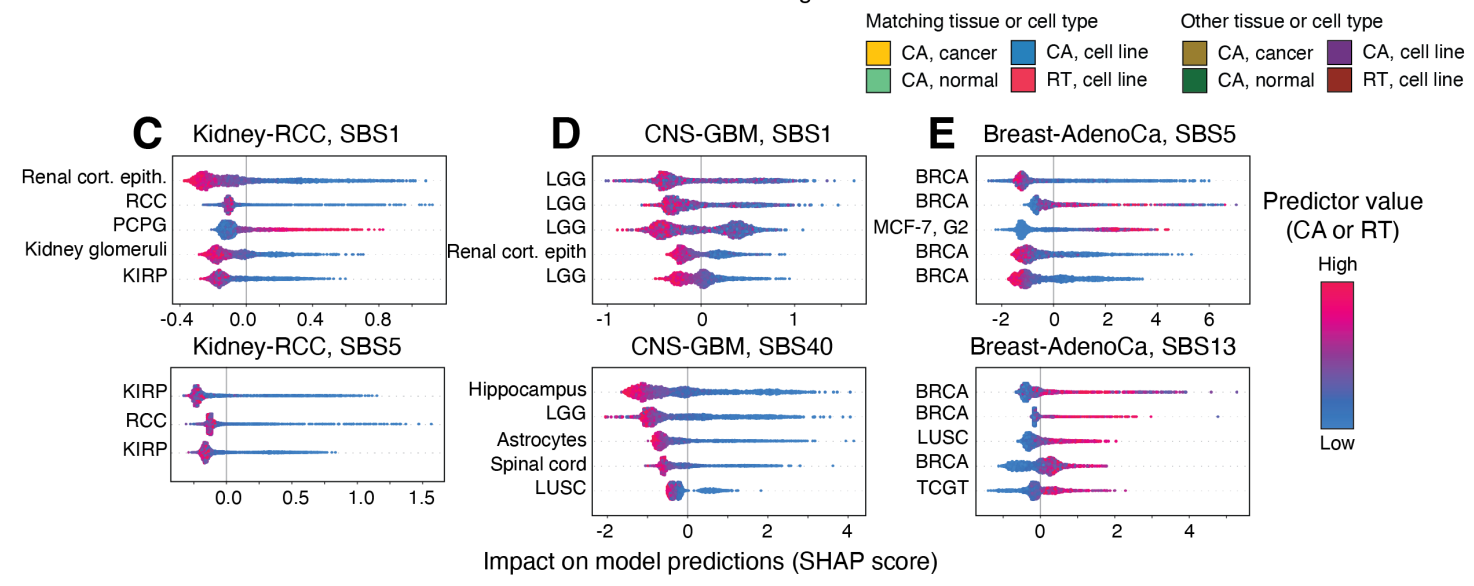

Figure 4. Associations of mutational signatures with chromatin accessibility and replication timing. A. Comparison of prediction accuracy of regional distribution of mutational signatures informed by CA and RT profiles. Signatures of carcinogens, unkown origin and SBS5,40 are more accurately predicted by CA and RT profiles than endogenous signatures. P-values adjust for genome-wide signature burden as covariate (F-test). B. CA and RT profiles associated with mutational signatures represent tissues of origin. Colored tiles show top-5 predictors of regional distribution of mutational signatures (permutation $P<0.001$ ). Colors indicate the predictor type $(C A, R T)$ and its relationship to the cancer type where mutagenesis is predicted (matching or other). Brighter colors indicate predictors that match cancer type or tissues or cells of origin. C-F. Examples of predictors of regional mutagenesis. Signatures are grouped vertically by etiology. Bulk mutation profiles are shown in the first row. SBS1 (2nd row) reveals a a diversity of predictors compared to other signatures. C-E. Examples of CA and RT profiles as predictors of mutational signatures. SHAP scores show the impact of a predictor on the predictions (X-axis) and corresponding predictor values (color gradient). C. In kidney cancer, SBS1 mutations (top) are best predicted by CA profiles of normal kidney cells and kidney cancers (RCC, KIRP) while only CA profiles of kidney cancers are the strongest predictors of SBS5 (bottom). D. In glioblastoma (GBM), SBS1 mutations (top) are best predicted by CA profiles of low-grade gliomas (LGG) while the strongest predictors of SBS40 (bottom) include CA profiles of normal neuronal tissues. E. In breast cancer, higher CA of BRCA primarily associates with fewer SBS5 mutations (top), while for SBS13 mutations, higher CA associates with higher burden (bottom). 


\section{Associations of mutational signatures with chromatin accessibility and replication timing}

183 We asked whether the associations of regional mutagenesis with CA and RT can be further

184 explained by mutational signatures. We assigned each SNV to its most probable single base

185 substitution (SBS) signature (6) and predicted the regional distributions of signatures using 773

186 CA and RT profiles. First, we compared the accuracy values of random forest models in

187 predicting six classes of mutational signatures based on etiology: two age-related classes (SBS1

188 and SBS5/40), APOBEC/AID, DNA-repair, and carcinogen signatures, and signatures of

189 unknown cause. Three classes of signatures were more informative of CA and RT profiles across

19014 cancer types (Figure 4A): predictions of carcinogenic signatures, signatures of unknown

191 cause, and aging-associated signatures (SBS5 and SBS40) were significantly more accurate than predictions of endogenous signatures of DNA repair, APOBEC/AID, and SBS1 (median adj. $\mathrm{R}^{2} \geq$ $0.62 v s$. adj. $\mathrm{R}^{2}=0.30$; F-test $P \leq 10^{-3}$ ), when accounting for total signature burden as a covariate of model accuracy. Thus, the mutational processes of carcinogen exposures, aging and unknown signatures show stronger interactions with CA and RT in cancer genomes.

We identified the top CA and RT profiles predictive of individual mutational signatures $(P<$ 0.001) (Figure 4B). Top predictors of signatures were often in agreement with those of bulk regional mutation burden: matching $\mathrm{CA}$ profiles of cancers were the top predictors of mutational signatures in breast, kidney, colorectal and stomach cancers, while RT profiles of normal cells associated with mutations in SCCs and lymphoid cancers. Top predictors of endogenous and exogeneous signatures were also mostly consistent, indicating that various mutational processes are affected by the epigenetic landscapes of cancers or cells of origin.

Signature SBS1 deviated from broad CA-driven patterns of regional mutagenesis in several cancer types. In kidney cancer, SBS1 mutations associated with CA profiles of normal tissues such as renal cortex epithelium and kidney glomerulus as well as extra-adrenal pheochromocytoma (PCPG), a rare endocrine cancer. In contrast, SBS5 mutations and others predominantly associated with CA of kidney cancers (Figure 4C). Similar effects were observed in breast, colorectal and stomach cancers: SBS1 mutations associated with CA profiles of normal tissues and unrelated cancers, while other signatures associated with CA profiles of matching cancers. However, SBS1 mutations were predicted less accurately than other signatures, potentially due to their overall lower frequency (Supplementary Figure 7). Interestingly, an inverse relationship was observed in GBM that may reflect its intratumoral heterogeneity and stem cell origins: the top predictors of SBS1 included CA profiles of four lower-grade gliomas (LGG) while the normal tissue profiles of hippocampus, astrocytes, and spinal cord primarily associated with clock-like signatures SBS5 and SBS40 (Figure 4D). The clock-like SBS1 signature of 5-methylcytosine deamination is associated with cancer patient age and stem cell division rate and affects the somatic genomes of normal tissues and adult stem cells $(10,35,36)$.

218 The association of SBS1 with the epigenomes of normal tissues suggests that SBS1 mutations in

219 cancer genomes represent a footprint of earlier cancer evolution or somatic mutagenesis in 220 normal cells of cancer origin. 
221 We asked whether the mutations of specific signatures were enriched or under-represented in 222 regions of open chromatin. While mutational signatures were generally negatively associated 223 with $\mathrm{CA}$ in accordance with bulk mutations, positive associations were also apparent. In breast 224 cancer, SBS13 mutations of APOBEC/AID activity positively associated with high CA scores 225 (Figure 4E), in agreement with the observations that AID targeting of epigenetically active 226 elements results in kataegis and clustered mutational signatures $(6,37,38)$. As another example, 227 SBS1 mutations in kidney cancer positively associated with the CA profile of the PCPG cancer 228 while negative associations with CA were apparent in other signatures and CA profiles (Figure 229 4C). In summary, this analysis highlights the complex interactions of CA and RT with regional 230 mutagenesis and cancer heterogeneity and helps characterize the mechanisms of mutational 231 processes. 
bioRxiv preprint doi: https://doi.org/10.1101/2021.05.14.444202; this version posted May 17, 2021. The copyright holder for this preprint (which was not certified by peer review) is the author/funder, who has granted bioRxiv a license to display the preprint in perpetuity. It is made available under aCC-BY 4.0 International license.

A

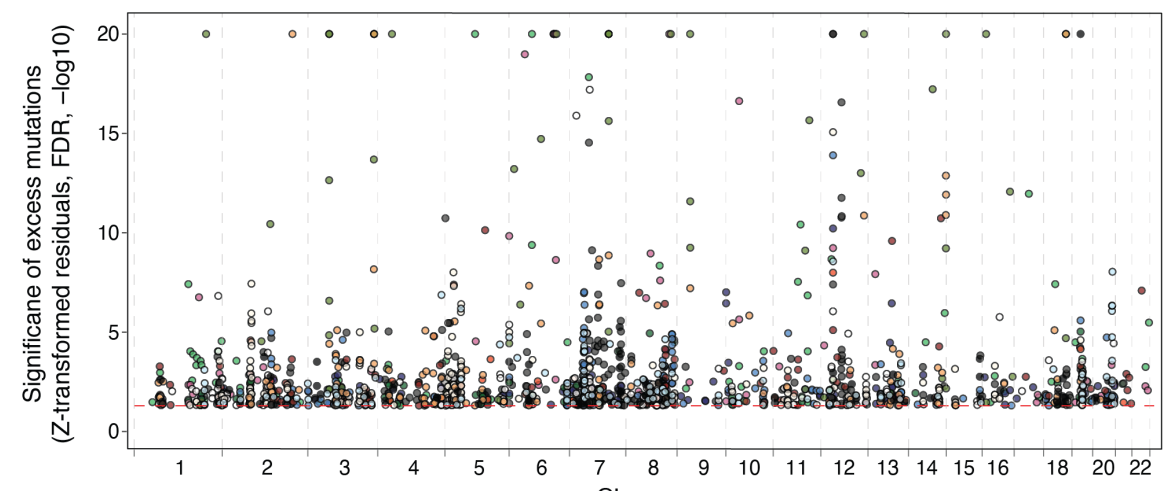

Cancer type

O Biliary-AdenoCa

- Breast-AdenoCa

- CNS-GBM

- ColoRect-AdenoCa

- Eso-AdenoCa

- Head-SCC

- Kidney-RCC

- Liver-HCC

Lung-AdenoCa

$O$ Lung-SCC

O Lymph-BNHL

O Lymph-CLL

- Skin-Melanoma

O Stomach-AdenoCa

B
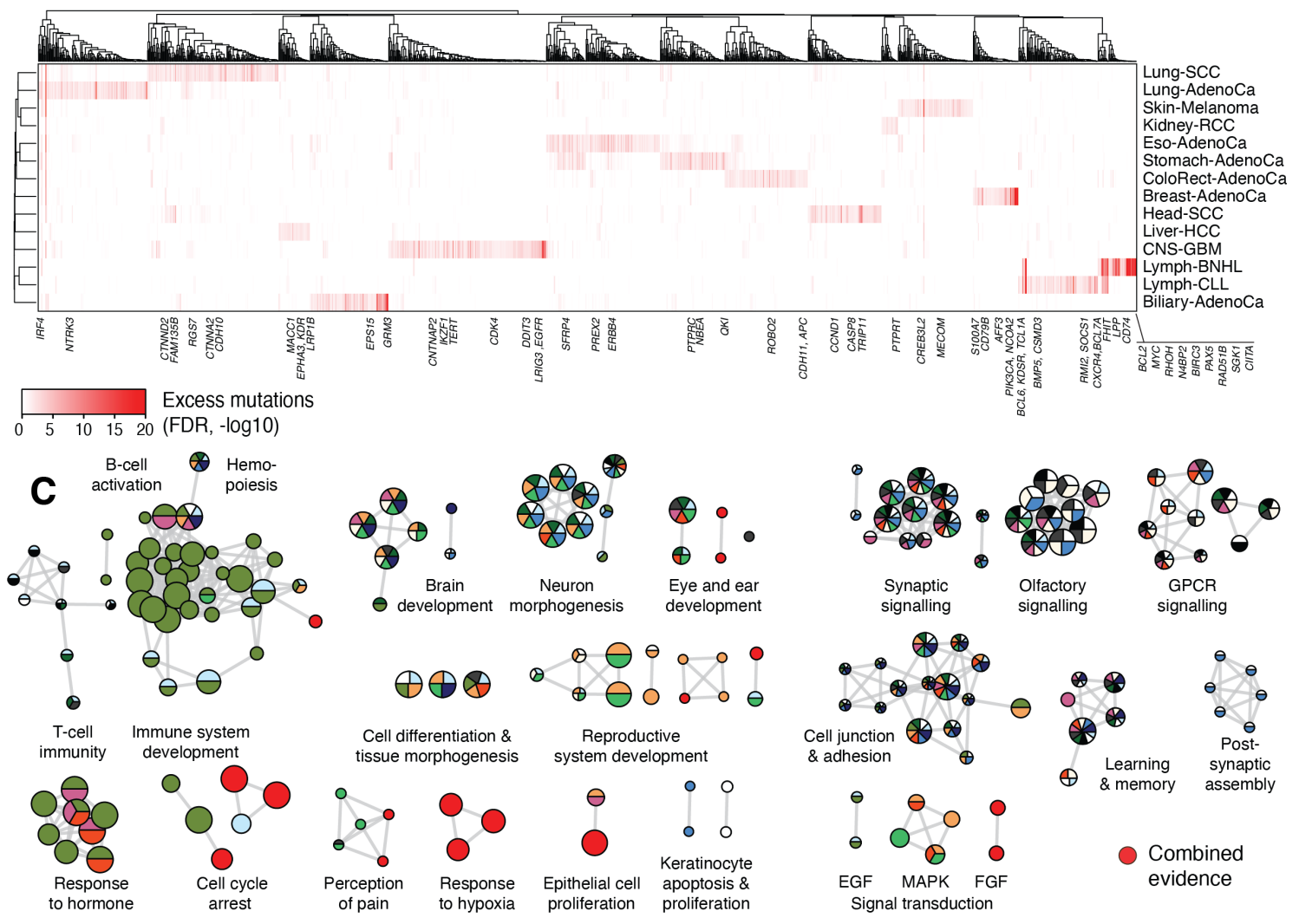

Figure 5. Excess mutations unexplained by epigenomes converge to cancer genes and developmental pathways. A. Manhattan plot shows the genomic regions (100 kbps) where the observed mutation frequencies exceed the predictions of CA and RT profiles in 14 cancer types. The significance of increased model residuals is shown on the $\mathrm{Y}$-axis (FDR $<0.05$; one-sided tests). B. Hierarchical clustering of frequently-mutated genomic regions shows the grouping of related cancer types and enrichment of known cancer genes (listed below). $\mathbf{C}$. Enrichment map of biological processes and pathways enriched in the genes of frequently-mutated regions (FDR $<0.05)$. Nodes represent pathways and edges connect pathways that share many genes. Nodes are grouped as subnetworks representing common biological themes. Colors show the cancer types where the pathway enrichments were detected (color legend in (A)). Node size corresponds to the number of genes in the pathway. Red nodes represent pathways that were only detected in the joint analysis of multiple cancer types. 
234 Excess mutations unexplained by epigenomes converge to cancer genes and developmental 235 pathways

236 To quantify the regional mutagenesis unexplained by CA and RT, we investigated the genomic

237 regions that were enriched in mutations above the levels expected from epigenomes. To enable a

238 gene-level functional analysis, we repeated the predictions of regional mutagenesis at a finer

239 genomic resolution ( $100 \mathrm{kbps})$ and selected 1,330 regions in 14 cancer types that were

240 significantly enriched in mutations based on the CA- and RT-informed model residuals $(F D R<$

241 0.05) (Figure 5A). While the mutation-enriched regions were largely tissue-specific with $86 \%$

242 detected in only one cancer type, hierarchical clustering of the regions by residuals was

243 consistent with cancer types (Figure 5B). For example, lymphoid cancers, lung cancers and

244 gastrointestinal cancers made up the three most distinct clusters. Thus, the regional mutational

245 processes independent of CA and RT affect similar genomic regions in related cancer types.

246 We performed a functional analysis of the frequently mutated genomic regions, hypothesizing

247 that these could be characterized by pathways and genes involved in cancer. The regions

248 encoded 730 protein-coding genes including 61 known cancer genes (39), significantly more

249 than expected by chance (27 expected, Fisher's exact $P=1.9 \times 10^{-9}$ ) (Figure 5B). Most driver

250 genes were only found in single cancer types and represented key disease-specific drivers such as

251 EGFR and TERT in glioma, MYC in BNHL, PIK3CA in breast cancer and APC in colorectal

252 cancer (Supplementary Figure 8). As an exception, one genomic window recurrently mutated

253 in 11 cancer types includes the interferon regulatory factor and oncogene IRF4 (40), the

254 phosphatase DUSP22 recently suggested as a network-implicated driver gene due to non-coding

255 mutations (41), and super-enhancers of immune cells (42), indicating a potential pan-cancer

256 region of interest (Supplementary Figure 9).

257 We then asked whether the frequently-mutated regions were associated with common biological

258 functions by prioritizing pan-cancer signals of mutation enrichment using the integrative

259 ActivePathways method (43). The analysis revealed 220 significantly enriched pathways $(F D R<$

260 0.05), of which 162 (74\%) were detected in more than one cancer type (Figure 5C).

261 Developmental processes including the nervous system, heart and kidney, stem cell development

262 and morphogenesis were prominently represented together with cancer hallmark processes such

263 as cell cycle, apoptosis, cell adhesion, hypoxia response, and MAPK, EGF and FGF signalling

264 pathways. Processes of the immune system, stress response, reproduction and hormone

265 regulation were also apparent. Enriched mutations converged to similar pathways and processes

266 across multiple cancer types although most genomic regions were only detected in one or few

267 cancer types. Convergence of these excess mutations to developmental and cancer pathways is

268 potentially explained by further mutational processes targeting active regions of the genome,

269 while the enrichment of known cancer driver genes suggests that positive selection of functional

270 mutations may also contribute to this additional mutation burden. This analysis exemplifies the

271 complex interplay of multi-scale mutational processes and genome function. 


\section{DISCUSSION}

274 Our analysis highlights chromatin accessibility of primary human cancers as a major covariate of

275 regional mutational processes that is supported by tissue of origin associations of whole cancer

276 genomes and epigenomes of matching cancer types. These observations are apparent in several

277 common cancer types of the largest global burden. Cancers such as melanoma and lymphoma

278 where normal tissue epigenomes are highly predictive of regional mutagenesis have an etiology

279 consistent with early somatic mutagenesis in normal tissues of origin. Combined associations

280 with normal and cancer epigenomes as observed in GBM may also reflect intratumoral

281 heterogeneity of cell populations and regional mutagenesis. These findings extend earlier studies

282 that used the epigenetic profiles of cell lines and normal tissues to characterize mutational

283 processes. Overall, this analysis suggests that in most cancer types, the megabase-scale

284 landscape of passenger mutations is primarily shaped later in cancer evolution following the

285 epigenetic transformation to cancer cells.

286 Replication timing information also associated with regional mutagenesis and confirmed strong

287 effects with cell types related to cancer origin. However, CA profiles of primary human cancers

288 evidently captured a larger fraction of variation of regional mutagenesis compared to RT

289 profiles, apart from squamous cell cancers that strongly associated with relevant cell lines. RT

290 profiles make up a smaller subset of epigenomic predictors in our dataset and include mitotic cell

291 lines that offer only limited representation of the diverse disease types in the pan-cancer cohort.

292 Interestingly, DNA replication has been shown to determine chromatin state (44). Thus, the

293 informative CA profiles of human cancers may represent a proxy of cancer-specific replication

294 dynamics.

295 Mutational signature analysis revealed interactions of mutational processes with CA and tissues

296 of origin. Carcinogen signatures, as well as signatures of unknown etiology, were overall better

297 predicted by CA and RT, in contrast to signatures of aging and DNA damage where the genome-

298 wide predictions were less accurate. The stronger association of carcinogen signatures suggests

299 that the chromatin environment interacts with DNA damage or repair processes of carcinogen

300 exposure, for example through elevated mutational processes targeting active genes that are

301 otherwise protected from mutations through error-free mismatch repair (38). Early replicating

302 regions in cells exposed to tobacco mutagens show elevated mutagenesis in transcribed strands

303 due to differential nucleotide excision repair activity (45). Based on their stronger interactions

304 with RT and CA profiles, we extrapolate that some mutational signatures of currently unknown

305 etiology may relate to carcinogens. SBS17a/b mutations show some of the strongest interactions

306 with CA and RT in stomach and esophageal cancers in our analysis. This signature is currently

307 of unknown cause, however it has been linked to gastric acid reflux and reactive oxygen species

308 (24). Further integrative analysis of clinical and lifestyle information with patterns of regional

309 mutagenesis may shed light to these mutational processes.

310 Mutations of signature SBS1 associated with CA profiles of relevant normal tissues in multiple

311 cancer types, in contrast to other signatures that were often associated with cancer epigenomes. 
312 SBS1 mutations follow a clock-like pattern whose frequency in cancer genomes correlates with 313 patient age and stem cell replication rates (35). SBS1 mutations contribute to the somatic

314 variation landscapes of normal adult tissues (46) and in embryonic development (47). Based on

315 our data, we speculate that SBS1 mutations in cancer genomes represent a footprint of early

316 cancer evolution or somatic variation of normal cells that occurs prior to the acquisition of

317 cancer-specific epigenetic profiles.

318 We observed a functional convergence to developmental processes and cancer-related pathways

319 in the genomic regions where CA and RT profiles insufficiently captured elevated mutation

320 burden. These data suggest that additional mutational processes affect lineage-specific

321 developmental genes and open-chromatin regions that are distinct in individual cancer types,

322 however map to the same molecular pathways across cancer types. For example, transcription

323 start sites of highly expressed genes and constitutively-bound binding sites of CTCF are subject

324 to elevated local mutagenesis in multiple cancer types (16). Lineage-specific genes are enriched

325 in indel mutations in solid cancers (48). Such local mutational processes confound the

326 observations at the megabase resolution of the genome where open chromatin is generally

327 associated with a lower mutation frequency. On the other hand, the enrichment of cancer genes

328 and pathways in our data suggests that some mutations unexplained by CA and RT are functional

329 in cancer and their frequent occurrence at specific genes, non-coding elements and molecular

330 pathways is explained by positive selection (3-5,41). Further study of these regions may deepen

331 our understanding of mutational processes and refine the catalogues of driver mutations.

332 This approach enables future studies to decipher the mechanisms and phenotypic associations of

333 mutational processes. Clinical, genetic, and epigenetic profiles of cancer patients can be

334 integrated to understand how regional mutational processes and the chromatin landscape are

335 modulated by clinical variables such as stage, grade or the therapies applied, genetic features

336 such as somatic driver mutations or inherited cancer risk variants, or lifestyle choices such as

337 tobacco or alcohol consumption. Complementary insights from sub-clonal reconstruction

338 analysis of cancer genomes $(2,49)$, as well as single-cell sequencing of genomes and epigenomes

339 will allow mapping of regional mutagenesis at the level of distinct cell populations contributing

340 to temporal and spatial variation in mutational processes. As such multimodal datasets grow, we

341 can learn about early cancer evolution by comparing regional mutagenesis in the genomes of

342 cancers and normal cells. Understanding the molecular and genetic determinants of regional

343 mutagenesis and signatures in cancer genomes may help characterize carcinogen exposures and

344 genetic predisposition, ultimately enhancing early cancer detection and prevention in the future. 
Methods

Somatic mutations in whole cancer genomes. Somatic single nucleotide variants (SNVs) of 2,583 whole cancer genomes of were derived from the Pan-cancer Analysis of Whole Genomes (PCAWG) project (1) and hypermutated tumors (66) were removed, resulting in a dataset of 23.2 million SNVs in 2,517 cancer genomes. Indel mutations and all variants in sex chromosomes were excluded. We analyzed 25 cancer types with at least 30 genomes per cohort and the pancancer cohort of 37 cancer types (Supplementary Figure 1). Mutations were mapped to GRCh38 coordinates using LiftOver (50).

Chromatin accessibility (CA) and replication timing (RT). 381 CA profiles of primary human cancers were retrieved from the TCGA study (27). 296 CA profiles of normal human tissues and cell lines were derived from ENCODE3 (28) and Epigenomics Roadmap (29) (Supplementary Figure 2). RT (RepliSeq) profiles of 16 human cell lines and 6 cell cycle phases were derived from the ENCODE study (30). CA and RT profiles were mapped to GRCh38 where needed.

Regional variation in mutation burden, CA, and RT. The genome was segmented into 2,465 distinct regions of one megabase (Mbps) after excluding sex chromosomes and filtering lowly mappable regions using the UMAP software (51). For each window, bulk SNV counts and SNV counts grouped by SBS signatures were derived for every cancer cohort. To create CA and RT profiles, mean values of each track were derived for every genomic region.

Random forest regression. Regional mutation burden was modeled as a function of CA and RT profiles with random forest regression (52). Monte-Carlo cross-validation was used evaluate model performance over $1,00080 / 20 \%$ data splits for training and validation. We used the adjusted $R^{2}$ (adj. $R^{2}$ ) metric of accuracy that measures the complexity-adjusted fraction of variance explained by the model.

368 CA of normal cells and cancers in regional mutagenesis. Two sets of random forest regression models with CA profiles of cancers and normal cells, respectively, were run in 1,000 MonteCarlo 80/20\% cross-validations using matched genomic regions for training and validation. Differences in adj. $\mathrm{R}^{2}$ values of models informed by cancer $\mathrm{CA}$ profiles relative to models informed by normal tissue CA profiles were computed ( $\Delta$ adj. $\mathrm{R}^{2}$ ) with $95 \%$ confidence intervals. Scatterplots of mutation counts were derived from models trained with full data.

Feature analysis of individual CA and RT profiles. The increase in mean-squared-error (incMSE) metric was used to evaluate CA and RT profiles as predictors of regional mutagenesis. Significance and variation of incMSE was evaluated. Permutation tests were used to detect the profiles where the incMSE values significantly exceeded those derived from randomly shuffled model responses. Empirical p-values were computed for every profile and cancer type across 1,000 iterations and significant profiles were selected $(P<0.001)$. Bootstrap analysis of genomic regions over 1,000 iterations revealed the variation in incMSE values.

381 Local effects of CA and RT profiles to regional mutagenesis. The Shapley Additive

382 exPlanation (SHAP) method (31) was used to evaluate the effects of profiles to mutagenesis in 
specific cancers and genomic regions. SHAP scores represent the importance and direction of each feature (i.e., a CA or RT profile) in predicting an observation (i.e., a genomic window). SHAP scores were computed separately for cancer types on models trained on full datasets.

Mutational signature analysis. SNVs were annotated to single base substitution (SBS) signatures from PCAWG (6) using top probabilities. In each cancer type, signatures with at least 10,000 mutations and at least 5\% of total mutation burden were selected (Supplementary Figure 7). Random forest regression was conducted with evaluation of accuracy and feature analysis as described above. Signatures were grouped based on etiology according to the COSMIC database (v 3.2, downloaded March 2021). Adj. $\mathrm{R}^{2}$ values for different groups of signatures were compared using ANOVA analysis and F-tests where the average signature exposures in cancer types were used as covariates. mutagenesis was predicted for 100-kbps genomic regions to enable more granular functional interpretation. Genomic regions were selected based on model residuals, i.e., where the observed mutation counts significantly exceeded model predictions. Residuals were Z-transformed and the one-tailed P-values were adjusted for multiple testing $(F D R<0.05)$. Regions with excess mutations were visualized as a heatmap with hierarchical clustering and correlation distance.

400 Known cancer genes were derived from the Cancer Gene Census database (39) (downloaded 401 Nov 26 $\left.{ }^{\text {th }} 2020\right)$. Enrichment of cancer genes was evaluated with a Fisher's exact test.

402 Pathway enrichment analysis of highly-mutated genomic regions. Integrative pathway 403 enrichment analysis with the ActivePathways method (43) was used to find overrepresented 404 pathways and prioritize genes. Genes were assigned the P-values of their genomic regions and 405 were scored in ActivePathways such that genes in regions with mutation enrichments in multiple 406 cancer types ranked higher. Significantly over-represented pathways $(F D R<0.05)$ were 407 visualized as an enrichment map using standard protocols (53).

408 A detailed description of methods is available in Supplementary Materials.

409 Acknowledgments. We thank Christian A. Lee, Kevin Cheng, Dr. Phedias Diamandis and Dr. 410 Anne Martel for constructive comments on this study. This work was supported by the Canadian 411 Institutes of Health Research (CIHR) Project Grant to J.R., A New Investigator Award of the 412 Terry Fox Research Institute (TFRI) to J.R., and the Investigator Award to J.R. from the Ontario 413 Institute for Cancer Research (OICR). Funding to OICR is provided by the Government of 414 Ontario. The results shown here are in whole or part based upon data generated by the TCGA 415 Research Network: https://www.cancer.gov/tcga. We acknowledge the contributions of the many 416 clinical networks of ICGC and TCGA who provided samples and data to PCAWG. We thank the 417 patients and their families for their participation in ICGC and TCGA projects.

418 Author contributions. O.O. analyzed the data and prepared the figures. J.R. and O.O. 419 interpreted the data and wrote the manuscript. J.R. conceived and supervised the project. The 420 authors reviewed and edited the manuscript and approved the final version. 


\section{References}

422 1. ICGC-TCGA Pan-Cancer Analysis of Whole Genomes Consortium. (2020) Pan-cancer analysis of whole genomes. Nature, 578, 82-93. 2,658 cancers. Nature, 578, 122-128.
2. Gerstung, M., Jolly, C., Leshchiner, I., Dentro, S.C., Gonzalez, S., Rosebrock, D., Mitchell, T.J., Rubanova, Y., Anur, P., Yu, K. et al. (2020) The evolutionary history of

3. Martincorena, I., Raine, K.M., Gerstung, M., Dawson, K.J., Haase, K., Van Loo, P., Davies, H., Stratton, M.R. and Campbell, P.J. (2017) Universal Patterns of Selection in Cancer and Somatic Tissues. Cell, 171, 1029-1041 e1021.

4. Rheinbay, E., Nielsen, M.M., Abascal, F., Wala, J.A., Shapira, O., Tiao, G., Hornshøj, H., Hess, J.M., Juul, R.I., Lin, Z. et al. (2020) Analyses of non-coding somatic drivers in 2,693 cancer whole genomes. Nature, 578, 102-111.

5. Zhu, H., Uuskula-Reimand, L., Isaev, K., Wadi, L., Alizada, A., Shuai, S., Huang, V., Aduluso-Nwaobasi, D., Paczkowska, M., Abd-Rabbo, D. et al. (2020) Candidate Cancer Driver Mutations in Distal Regulatory Elements and Long-Range Chromatin Interaction Networks. Mol Cell.

6. Alexandrov, L.B., Kim, J., Haradhvala, N.J., Huang, M.N., Tian Ng, A.W., Wu, Y., Boot, A., Covington, K.R., Gordenin, D.A., Bergstrom, E.N. et al. (2020) The repertoire of mutational signatures in human cancer. Nature, 578, 94-101.

7. Li, Y., Roberts, N.D., Wala, J.A., Shapira, O., Schumacher, S.E., Kumar, K., Khurana, E., Waszak, S., Korbel, J.O., Haber, J.E. et al. (2020) Patterns of somatic structural variation in human cancer genomes. Nature, 578, 112-121.

8. Kumar, S., Warrell, J., Li, S., McGillivray, P.D., Meyerson, W., Salichos, L., Harmanci, A., Martinez-Fundichely, A., Chan, C.W.Y., Nielsen, M.M. et al. (2020) Passenger Mutations in More Than 2,500 Cancer Genomes: Overall Molecular Functional Impact and Consequences. Cell, 180, 915-927 e916.

9. Martincorena, I., Roshan, A., Gerstung, M., Ellis, P., Van Loo, P., McLaren, S., Wedge, D.C., Fullam, A., Alexandrov, L.B., Tubio, J.M. et al. (2015) Tumor evolution. High burden and pervasive positive selection of somatic mutations in normal human skin. Science, 348, 880-886.

10. Blokzijl, F., de Ligt, J., Jager, M., Sasselli, V., Roerink, S., Sasaki, N., Huch, M., Boymans, S., Kuijk, E., Prins, P. et al. (2016) Tissue-specific mutation accumulation in human adult stem cells during life. Nature, 538, 260-264.

11. Supek, F. and Lehner, B. (2019) Scales and mechanisms of somatic mutation rate variation across the human genome. DNA Repair (Amst), 102647.

12. Gonzalez-Perez, A., Sabarinathan, R. and Lopez-Bigas, N. (2019) Local Determinants of the Mutational Landscape of the Human Genome. Cell, 177, 101-114.

13. Pich, O., Muinos, F., Lolkema, M.P., Steeghs, N., Gonzalez-Perez, A. and Lopez-Bigas, N. (2019) The mutational footprints of cancer therapies. Nature genetics, 51, 1732-1740.

14. Katainen, R., Dave, K., Pitkanen, E., Palin, K., Kivioja, T., Valimaki, N., Gylfe, A.E., Ristolainen, H., Hanninen, U.A., Cajuso, T. et al. (2015) CTCF/cohesin-binding sites are frequently mutated in cancer. Nature genetics, 47, 818-821.

15. Sabarinathan, R., Mularoni, L., Deu-Pons, J., Gonzalez-Perez, A. and Lopez-Bigas, N. (2016) Nucleotide excision repair is impaired by binding of transcription factors to DNA. Nature, 532, 264-267. 
16. Lee, C.A., Abd-Rabbo, D. and Reimand, J. (2021) Functional and genetic determinants of mutation rate variability in regulatory elements of cancer genomes. Genome Biol, 22, 133.

17. Lawrence, M.S., Stojanov, P., Polak, P., Kryukov, G.V., Cibulskis, K., Sivachenko, A., Carter, S.L., Stewart, C., Mermel, C.H., Roberts, S.A. et al. (2013) Mutational heterogeneity in cancer and the search for new cancer-associated genes. Nature, 499, 214-218.

18. Schuster-Bockler, B. and Lehner, B. (2012) Chromatin organization is a major influence on regional mutation rates in human cancer cells. Nature, 488, 504-507.

19. Stamatoyannopoulos, J.A., Adzhubei, I., Thurman, R.E., Kryukov, G.V., Mirkin, S.M. and Sunyaev, S.R. (2009) Human mutation rate associated with DNA replication timing. Nature genetics, 41, 393-395.

20. Supek, F. and Lehner, B. (2015) Differential DNA mismatch repair underlies mutation rate variation across the human genome. Nature, 521, 81-84.

21. Zheng, C.L., Wang, N.J., Chung, J., Moslehi, H., Sanborn, J.Z., Hur, J.S., Collisson, E.A., Vemula, S.S., Naujokas, A., Chiotti, K.E. et al. (2014) Transcription restores DNA repair to heterochromatin, determining regional mutation rates in cancer genomes. Cell Rep, 9, 1228-1234.

22. Woo, Y.H. and Li, W.H. (2012) DNA replication timing and selection shape the landscape of nucleotide variation in cancer genomes. Nat Commun, 3, 1004.

23. Liu, L., De, S. and Michor, F. (2013) DNA replication timing and higher-order nuclear organization determine single-nucleotide substitution patterns in cancer genomes. Nat Commun, 4, 1502.

24. Tomkova, M., Tomek, J., Kriaucionis, S. and Schuster-Bockler, B. (2018) Mutational signature distribution varies with DNA replication timing and strand asymmetry. Genome Biol, 19, 129.

25. Jiao, W., Atwal, G., Polak, P., Karlic, R., Cuppen, E., Subtypes, P.T., Clinical Translation Working, G., Danyi, A., de Ridder, J., van Herpen, C. et al. (2020) A deep learning system accurately classifies primary and metastatic cancers using passenger mutation patterns. Nat Commun, 11, 728.

26. Polak, P., Karlic, R., Koren, A., Thurman, R., Sandstrom, R., Lawrence, M.S., Reynolds, A., Rynes, E., Vlahovicek, K., Stamatoyannopoulos, J.A. et al. (2015) Cell-of-origin chromatin organization shapes the mutational landscape of cancer. Nature, 518, 360-364.

27. Corces, M.R., Granja, J.M., Shams, S., Louie, B.H., Seoane, J.A., Zhou, W., Silva, T.C., Groeneveld, C., Wong, C.K., Cho, S.W. et al. (2018) The chromatin accessibility landscape of primary human cancers. Science, $\mathbf{3 6 2}$.

28. Vierstra, J., Lazar, J., Sandstrom, R., Halow, J., Lee, K., Bates, D., Diegel, M., Dunn, D., Neri, F., Haugen, E. et al. (2020) Global reference mapping of human transcription factor footprints. Nature, 583, 729-736.

29. Roadmap Epigenomics Consortium, Kundaje, A., Meuleman, W., Ernst, J., Bilenky, M., Yen, A., Heravi-Moussavi, A., Kheradpour, P., Zhang, Z., Wang, J. et al. (2015) Integrative analysis of 111 reference human epigenomes. Nature, 518, 317-330.

30. Hansen, R.S., Thomas, S., Sandstrom, R., Canfield, T.K., Thurman, R.E., Weaver, M., Dorschner, M.O., Gartler, S.M. and Stamatoyannopoulos, J.A. (2010) Sequencing newly replicated DNA reveals widespread plasticity in human replication timing. Proc Natl Acad Sci U S A, 107, 139-144. 
31. Lundberg, S.M., Erion, G., Chen, H., DeGrave, A., Prutkin, J.M., Nair, B., Katz, R., Himmelfarb, J., Bansal, N. and Lee, S.I. (2020) From Local Explanations to Global Understanding with Explainable AI for Trees. Nat Mach Intell, 2, 56-67.

32. Marino, S. (2005) Medulloblastoma: developmental mechanisms out of control. Trends Mol Med, 11, 17-22.

33. Odegard, V.H. and Schatz, D.G. (2006) Targeting of somatic hypermutation. Nat Rev Immunol, 6, 573-583.

34. Singh, S.K., Hawkins, C., Clarke, I.D., Squire, J.A., Bayani, J., Hide, T., Henkelman, R.M., Cusimano, M.D. and Dirks, P.B. (2004) Identification of human brain tumour initiating cells. Nature, 432, 396-401.

35. Alexandrov, L.B., Jones, P.H., Wedge, D.C., Sale, J.E., Campbell, P.J., Nik-Zainal, S. and Stratton, M.R. (2015) Clock-like mutational processes in human somatic cells. Nature genetics, 47, 1402-1407.

36. Abascal, F., Harvey, L.M.R., Mitchell, E., Lawson, A.R.J., Lensing, S.V., Ellis, P., Russell, A.J.C., Alcantara, R.E., Baez-Ortega, A., Wang, Y. et al. (2021) Somatic mutation landscapes at single-molecule resolution. Nature.

37. Wang, Q., Oliveira, T., Jankovic, M., Silva, I.T., Hakim, O., Yao, K., Gazumyan, A., Mayer, C.T., Pavri, R., Casellas, R. et al. (2014) Epigenetic targeting of activationinduced cytidine deaminase. Proc Natl Acad Sci U S A, 111, 18667-18672.

38. Supek, F. and Lehner, B. (2017) Clustered Mutation Signatures Reveal that Error-Prone DNA Repair Targets Mutations to Active Genes. Cell, 170, 534-547 e523.

39. Futreal, P.A., Coin, L., Marshall, M., Down, T., Hubbard, T., Wooster, R., Rahman, N. and Stratton, M.R. (2004) A census of human cancer genes. Nat Rev Cancer, 4, 177-183.

40. Iida, S., Rao, P.H., Butler, M., Corradini, P., Boccadoro, M., Klein, B., Chaganti, R.S. and Dalla-Favera, R. (1997) Deregulation of MUM1/IRF4 by chromosomal translocation in multiple myeloma. Nature genetics, 17, 226-230.

41. Reyna, M.A., Haan, D., Paczkowska, M., Verbeke, L.P.C., Vazquez, M., Kahraman, A., Pulido-Tamayo, S., Barenboim, J., Wadi, L., Dhingra, P. et al. (2020) Pathway and network analysis of more than 2,500 whole cancer genomes. Nature Communications, 11, 729.

42. Hnisz, D., Abraham, B.J., Lee, T.I., Lau, A., Saint-Andre, V., Sigova, A.A., Hoke, H.A. and Young, R.A. (2013) Super-enhancers in the control of cell identity and disease. Cell, 155, 934-947.

43. Paczkowska, M., Barenboim, J., Sintupisut, N., Fox, N.S., Zhu, H., Abd-Rabbo, D., Mee, M.W., Boutros, P.C., PCAWG Drivers and Functional Interpretation Working Group, Reimand, J. et al. (2020) Integrative pathway enrichment analysis of multivariate omics data. Nature Communications, 11, 735.

44. Klein, K.N., Zhao, P.A., Lyu, X., Sasaki, T., Bartlett, D.A., Singh, A.M., Tasan, I., Zhang, M., Watts, L.P., Hiraga, S.I. et al. (2021) Replication timing maintains the global epigenetic state in human cells. Science, 372, 371-378.

45. Kucab, J.E., Zou, X., Morganella, S., Joel, M., Nanda, A.S., Nagy, E., Gomez, C., Degasperi, A., Harris, R., Jackson, S.P. et al. (2019) A Compendium of Mutational Signatures of Environmental Agents. Cell, 177, 821-836 e816.

46. Moore, L., Cagan, A., Coorens, T., Neville, M.D., Sanghvi, R., Sanders, M.A., Oliver, T.R., Leongamornlert, D., Ellis, P. and Noorani, A. (2020) The mutational landscape of human somatic and germline cells. bioRxiv. 
47. Bizzotto, S., Dou, Y., Ganz, J., Doan, R.N., Kwon, M., Bohrson, C.L., Kim, S.N., Bae, T., Abyzov, A., Network, N.B.S.M. et al. (2021) Landmarks of human embryonic development inscribed in somatic mutations. Science, 371, 1249-1253.

48. Imielinski, M., Guo, G. and Meyerson, M. (2017) Insertions and Deletions Target Lineage-Defining Genes in Human Cancers. Cell, 168, 460-472 e414.

49. Dentro, S.C., Leshchiner, I., Haase, K., Tarabichi, M., Wintersinger, J., Deshwar, A.G., Yu, K., Rubanova, Y., Macintyre, G., Demeulemeester, J. et al. (2021) Characterizing genetic intra-tumor heterogeneity across 2,658 human cancer genomes. Cell.

50. Lawrence, M., Gentleman, R. and Carey, V. (2009) rtracklayer: an R package for interfacing with genome browsers. Bioinformatics, 25, 1841-1842.

51. Karimzadeh, M., Ernst, C., Kundaje, A. and Hoffman, M.M. (2018) Umap and Bismap: quantifying genome and methylome mappability. Nucleic Acids Res, 46, e120.

52. Ho, T.K. (1995) Random decision forests. IEEE Proceedings of 3rd international conference on document analysis and recognition, 1, 278-282.

53. Reimand, J., Isserlin, R., Voisin, V., Kucera, M., Tannus-Lopes, C., Rostamianfar, A., Wadi, L., Meyer, M., Wong, J., Xu, C. et al. (2019) Pathway enrichment analysis and visualization of omics data using g:Profiler, GSEA, Cytoscape and EnrichmentMap. Nat Protoc, 14, 482-517. 